\title{
Position Control Mathematical Modelling and Operational Evaluation of Tele-operated Electro-hydraulic Actuator (T-EHA)
}

\author{
Ahmad Anas Yusof ${ }^{1, ~ a ~, ~ M o h d ~ N o o r ~ A s r i l ~ S a a d u n ~}{ }^{2, b}$, \\ Mohd Khairi Mohamed Nor ${ }^{3, \mathrm{c}}$, Mohd Qadafie Ibrahim ${ }^{4, \mathrm{~d}}$ \\ and Muhammad Zaidan Abdul Manaf ${ }^{5, e}$ \\ ${ }^{1,2,3}$ Faculty of Mechanical Engineering, \\ ${ }^{4,5}$ Faculty of Engineering Technology, \\ Universiti Teknikal Malaysia Melaka, Hang Tuah Jaya, 76100 Durian Tunggal, Melaka \\ aanas@utem.edu.my, basril@utem.edu.my, 'khairi@utem.edu.my, dqadafie@utem.edu.my , \\ ezaidan@utem.edu.my
}

Keywords: Tele-operation, Electro-Hydraulic Actuator, Position Control

Abstract. This study deals with a master-slave system for a tele-operated electro-hydraulic actuator (T-EHA) that focuses on a hydraulic system to remotely control a mini excavator. Tele-operation using such system is useful for tele-operation support of heavy construction and road restoration, typically in post-disaster areas. This paper presents the current development of position control electro-hydraulic actuator for such remote tele-operation application. A $2.4 \mathrm{GHz}$ radio-controlled transmitter and receiver unit, which is also known as the master, has been utilized as the remote controller for an electro-hydraulic actuator. The electro-hydraulic actuator, which serves as the slave has been fabricated by using a tie-rod cylinder, and coupled with a 24 VDC electro-hydraulic valve. Position control mathematical modelling and operational evaluation have been studied with regard to the tele-operated electro-hydraulic actuator.

\section{Introduction}

Tele-operated construction machinery is very useful under an extreme environment, such as in nuclear post-disaster recovery activities [1]. The tele-operation system is usually supported by a remote-controlled fluid power actuator, as used in the Robo-Q and PARM system [2,3]. In this paper, the actuator is modelled as handling a moving load, as shown in Fig. 1.

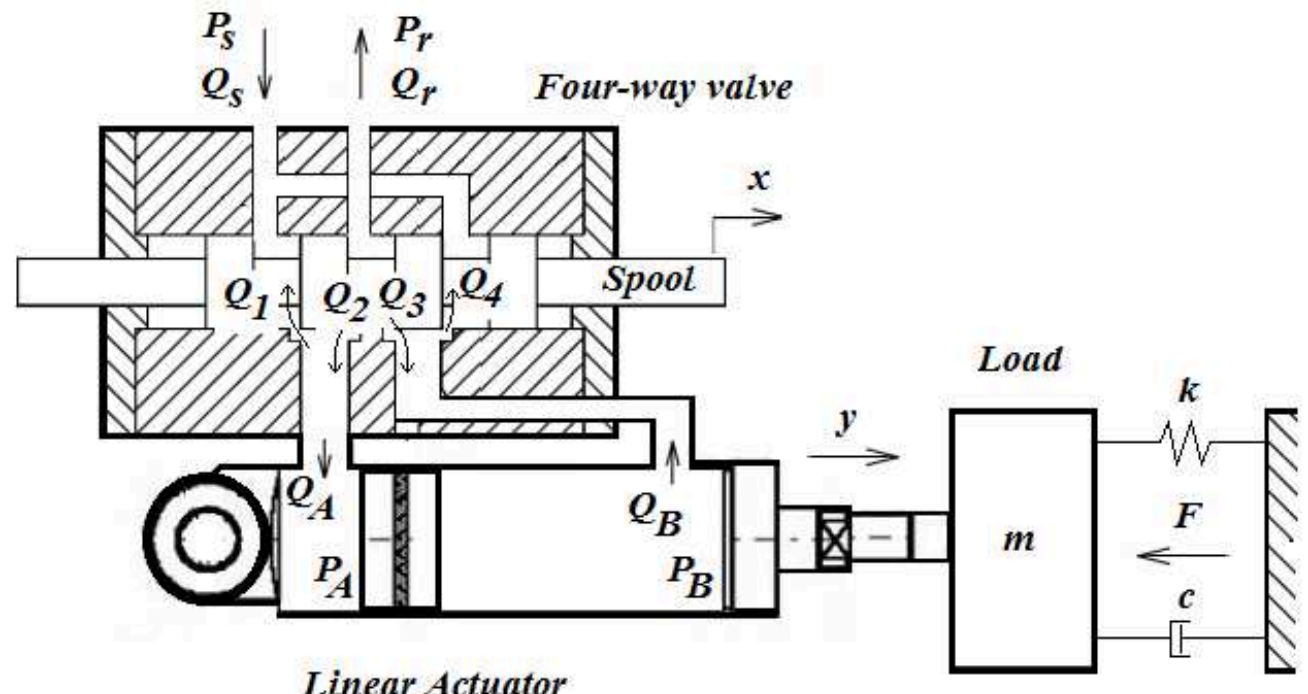

Fig.1 Tele-operated Electro-hydraulic Actuator, T-EHA (Slave Side) 
The T-EHA is actually represented as a single mass-spring-damper system with a load-disturbance force given by $F$. The mass, spring rate and viscous-drag coefficient for the load are represented by the symbols $m, k$, and $c$, respectively. The single rod linear actuator is connected to both the load and the actuator piston. The displacement of the spool valve $x$ is shown to be positive in the right direction. When the valve is moved in the positive $x$ direction, hydraulic fluid is directed into side $A$ of the actuator, which then requires the fluid to exit the actuator from side $B$. The flow rates are represented by the symbols $Q_{A}$ and $Q_{B}$, respectively. The flow is provided by a pump with volumetric displacement $V_{D}$, and driven by an electric motor, at rotational speed $N$. In the mathematical modelling, the resulted pressure transients from fluid compressibility are assumed to be minimum and therefore negligible. This is true for T-EHA design that involves a very short transmission lines between the valve and the actuator [4].

\section{Position Control Mathematical Modelling and Design}

The design of the T-EHA can be divided into master side and slave side, as shown in Fig. 2 . A 2.4 $\mathrm{GHz}$ radio-controlled transmitter and receiver unit has been utilized as the remote controller (also known as the master) of an electro-hydraulic actuator. The electro-hydraulic actuator, (which serves as the slave) has been fabricated by using a tie-rod cylinder, and coupled with a 24 VDC four- way electro-hydraulic valve. The tested operational range of the system is up to a 100 meters, so far.

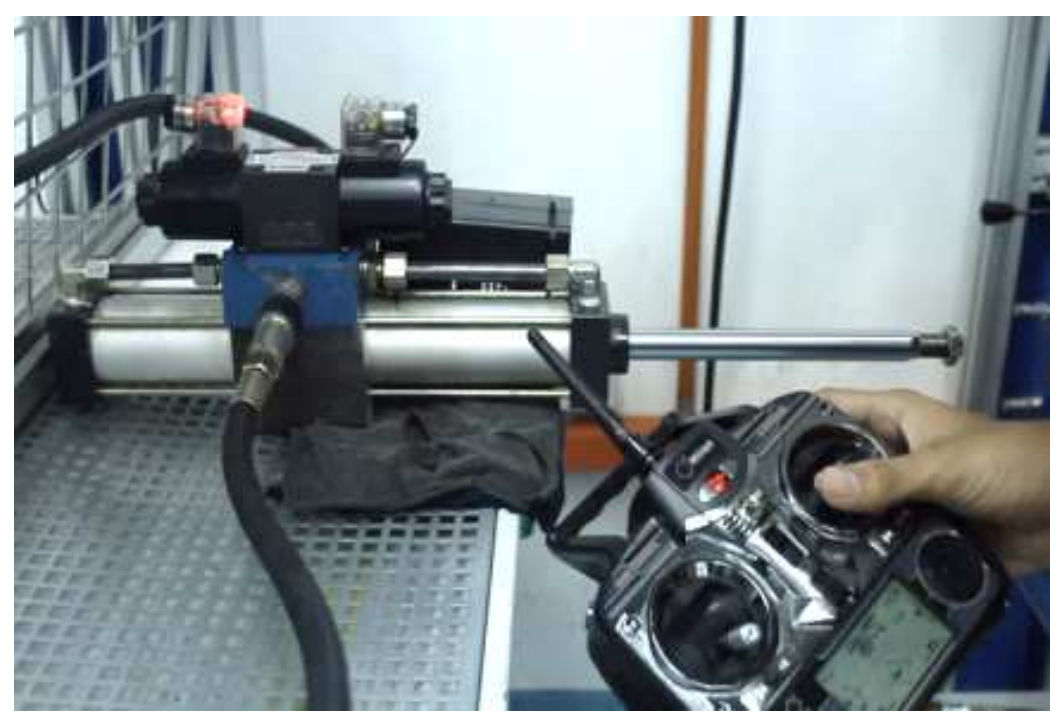

Fig. 2 The Fabricated T-EHA

The study on T-EHA starts with the basic loading analysis. The inertia of the actuator is neglected in the study. The value is much smaller compared to the actual forces that are generated by the actuator. The equation of motion for the load can be represented by [5],

$$
m \ddot{y}+c \dot{y}+k y=\eta_{a f}\left(A_{A} P_{A}-A_{B} P_{B}\right)-F-F_{o},
$$

where $P_{A}$ and $P_{B}$ are the fluid pressures on the $A$ and $B$ sides of the actuator, respectively. The actuator force efficiency is represented by $\eta_{a f} . F_{o}$ is the nominal spring or bias load that is applied to the actuator when $y$ equals zero. By assuming nominal steady state operating conditions, $(y=0$, $P_{A}=P_{B}=P_{S} / 2$, and $F=0$ ), it can be shown that during steady state conditions, the nominal force exerted on the actuator by the load is given as,

$$
F_{o}=\eta_{a f}\left(A_{A}-A_{B}\right) P_{s} / 2 \text {. }
$$


By substituting Eq.1 and Eq. 2, the following Eq. 3 is produced,

$$
m \ddot{y}+c \dot{y}+k y=\eta_{a f}\left(A_{A} P_{A}-A_{B} P_{B}\right)-\left(\eta_{a f}\left(A_{A}-A_{B}\right) P_{s} / 2\right)-F .
$$

Thus, the following Eq. 4 can be used to describe the output dynamics of the load,

$$
m \ddot{y}+c \dot{y}+k y=\eta_{a f}\left(A_{A}\left(P_{A}-P_{s} / 2\right)-A_{B}\left(P_{B}-P_{s} / 2\right)\right)-F .
$$

The equation shows that the actuator pressures $P_{A}$ and $P_{B}$ are the required input for adjusting the position of the load. The changing resistance to the flow going in and out of the T-EHA due to the loading adjustment will require actuator pressures to be increasing and decreasing accordingly. The changes can be described by the volumetric flow rates in and out on sides $A$ and $B$ of the T-EHA. The flow going in and out at side $\mathrm{A}$ is given as,

$$
Q_{A}=\frac{A_{A}}{\eta_{a v}} \dot{y},
$$

while the flow going through side B can be represented by,

$$
Q_{B}=\frac{A_{B}}{\eta_{a v}} \dot{y}
$$

where $Q_{A}$ and $Q_{B}$ are the volumetric flow rates into and out of the T-EHA, respectively, and $\eta_{a v}$ is the volumetric efficiency of the actuator. In this research, it is assumed that there is not much different for area $A_{A}$ and $A_{B}$, which will simplify the overall mathematical modelling of the TEHA. The volumetric flow rates of $Q_{A}$ can be represented at the spool valve in as,

$$
Q_{A}=Q_{2}-Q_{1},
$$

and for $Q_{B}$, the flow can be represented as,

$$
Q_{B}=Q_{4}-Q_{3}
$$

The linearized flow equations for fluid passing across the spool valve are given by,

$$
\begin{aligned}
& Q_{1}=K_{c} P_{s} / 2-K_{q} x+K_{c}\left(P_{A}-P_{r}\right) \\
& Q_{2}=K_{c} P_{s} / 2-K_{q} x+K_{c}\left(P_{s}-P_{A}\right) \\
& Q_{3}=K_{c} P_{s} / 2-K_{q} x+K_{c}\left(P_{s}-P_{B}\right) \\
& Q_{4}=K_{c} P_{s} / 2-K_{q} x+K_{c}\left(P_{B}-P_{r}\right)
\end{aligned}
$$

where $K_{c} P_{s}$ is the nominal flow rate across each the valve at the steady operating conditions of the system, and $K_{q}$ and $K_{c}$ are the flow gain and the pressure-flow coefficient for the valve, respectively. By substituting Eq. 7, Eq. 8 and Eq. 9, the volumetric flow rates into and out of the actuator may be expressed, respectively, as,

$$
\begin{aligned}
& Q_{A}=2 K_{q} x-2 K_{c}\left(P_{A}-P_{s} / 2\right) \\
& Q_{B}=2 K_{q} x-2 K_{c}\left(P_{B}-P_{s} / 2\right)
\end{aligned}
$$

where the return pressure $P_{r}=0$. Using this result with Eq. 5 and Eq. 6, the following equations for the operating pressures on both sides of the linear actuator may be written as, 


$$
\begin{aligned}
& P_{A}-P_{s} / 2=K_{p} x-\frac{A_{A}}{2 K_{c} \eta_{a v}} \dot{y} \\
& P_{B}-P_{S} / 2=-K_{p} x+\frac{A_{B}}{2 K_{c} \eta_{a v}} \dot{y}
\end{aligned}
$$

where $K_{p}$ is the valve's pressure sensitivity given by the ratio of the flow gain to the pressure-flow coefficient $K_{q} / K_{c}$. From Eq. 11, it can be seen that the fluid pressure on side $A$ of the linear actuator is increased by moving the spool valve in the positive $x$ direction and that the fluid pressure on side $B$ of the actuator is decreased by the same valve motion. By substituting Eq. 11 into Eq. 4, the following equation for the motion of the system is,

$$
m \ddot{y}+\left(c+\frac{A_{A}^{2}+A_{B}^{2}}{2 K_{c}}\right) \dot{y}+k y=\eta_{a f}\left(A_{A}+A_{B}\right) K_{p} x-F .
$$

The above equation shows that the mechanical design of the linear actuator and the spool valve have a significant influence on the overall dynamics of the T-EHA. The design parameters create an effective damping effect for the system, and provide adequate gain relationship between the input motion of the spool valve and the output motion of the load. The T-EHA is used to undertake accurate positioning of the load at the required actuator stroke within the operating range of the teleoperation. Such position-controlled tele-operation will require the operator to rely on the feedback displacement of the actuator [6,7]. At this moment, the operational evaluation is based on direct visual feedback during tele-operation $[8,9,10]$. By neglecting the inertia and viscous damping, Eq. 12 may be simplified as follows,

$$
k y=\eta_{a f}\left(A_{A}+A_{B}\right) K_{p} x-F
$$

where the block diagram representing the equation of the position control are shown in Fig. 3 .

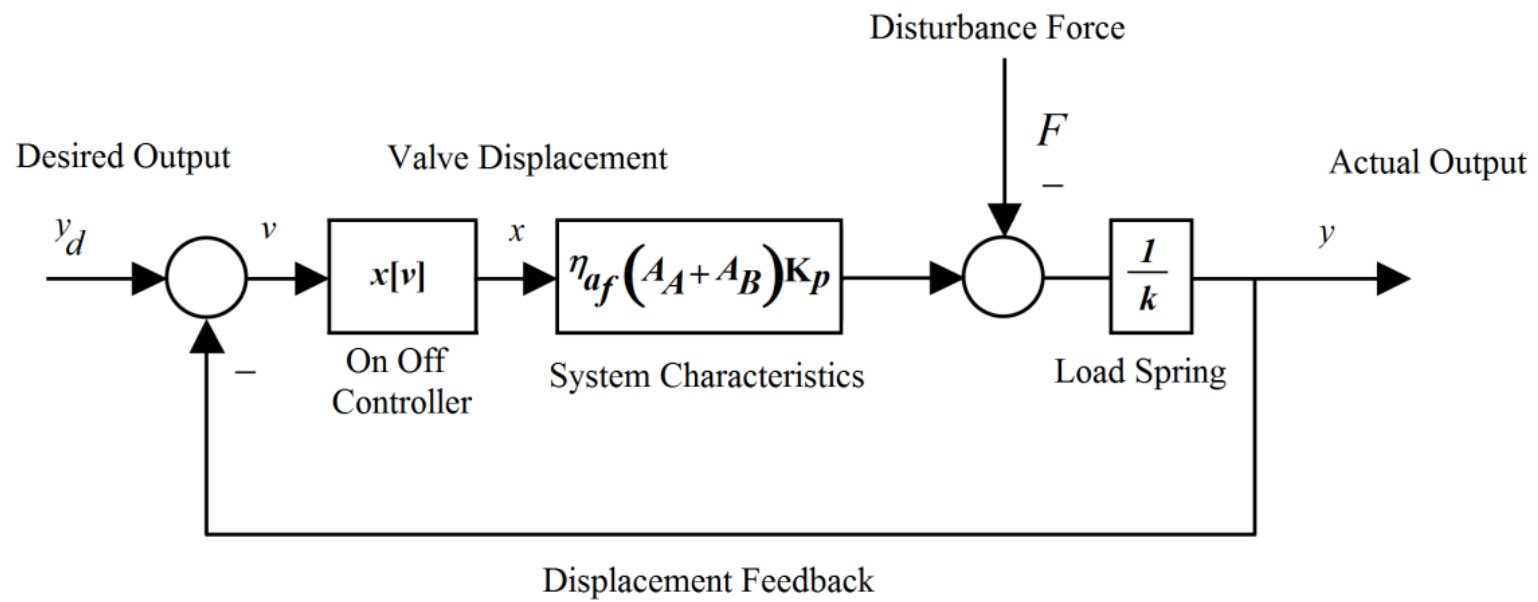

Fig. 3 The Position-Control Block Diagram of T-EHA

By using a standard on-off controller, the control law for the displacement of the valve can be written as,

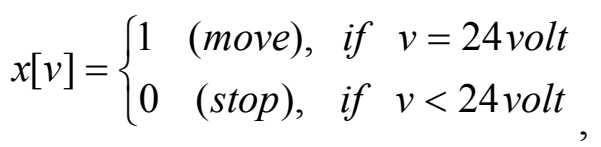

where $v$ represents the input voltage on the T-EHA. 


\section{Conclusion}

The development of position control tele-operated electro-hydraulic actuator (T-EHA) for remote tele-operation system for a mini-excavator is being conducted at the Centre for Advanced Research on Energy, Universiti Teknikal Malaysia Melaka. The objective of the study is to fabricate an integrated system of $2.4 \mathrm{GHz}$ radio controlled transmitter unit and electro-hydraulic actuator. The unit will serve as remote-controlled master-slave system. The platform will also be used to provide variety information regarding to the use of energy efficient solar system in powering the T-EHA on a construction machine in remote area. Preliminary position control mathematical modelling and the operational evaluation has been presented in the paper.

\section{Acknowledgments}

The research work is supported under the short term project PJP/2014/FKM(14A)/S01347. The authors wish to thank Universiti Teknikal Malaysia Melaka for their financial support.

\section{References}

[1] K. Chayama and K. Kenji "Fluid Power System in Post-Disaster Recovery", J. of The Japan Fluid Power System Society. 36(2005) 42-46.

[2] T. Sasaki and K. Kawashima, "Remote Control of Backhoe at Construction Site with a Pneumatic Robot System”, Automation in Construction. 17(2008) 907-914.

[3] H. Yamada, H. Kato, and T. Muto, Master-slave Control for Construction Robot Teleoperation, J. of Robotics and Mechatronics. 15(2003) 534-540.

[4] A. Akers, M. Gassman, and R. Smith, Hydraulic Power System Analysis. CRC Press, Boca Raton, 2006.

[5] N.D. Manring, Hydraulic Control System. John Wiley \& Sons, Inc., New York, 2005.

[6] A.A. Yusof, T. Kawamura, and H. Yamada, Evaluation of Construction Robot Tele-grasping Force Perception Using Visual, Auditory and Force Feedback Integration, J. of Robotics and Mechatronics. 24:6(2012) 949-957.

[7] L. Huang, T. Kawamura, and H. Yamada, Construction Robot Operation System with Object's Hardness Recognition Using Force Feedback and Virtual Reality J. of Robotics and Mechatronics.24:6 (2012)958-966.

[8] C. Preusche, and G. Hirzinger, Haptics in Tele-robotics: Current and Future Research and Applications, Visual Comput. 23 (2007) 273-284.

[9] A.M Okamura, Methods for Haptic Feedback in Teleoperated Robot-assisted Surgery. Industrial Robot: An Industrial Journal. 31 (2004) 499-508.

[10] M.E. Hagen, J.J.Meehan, I. Inan, and P. Morel, Visual Clues act as a Substitute for Haptic Feedback in Robotic Surgery. 22 (2008) 1505-1508. 